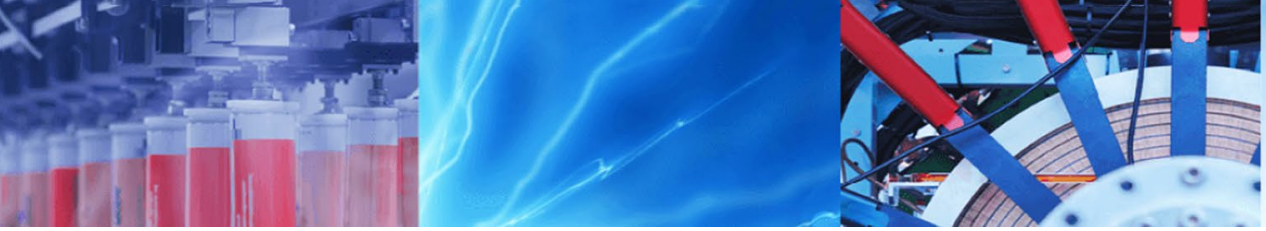

Research Article

\title{
Theory and implementation of the multiple valued iterative dynamics algorithms for a class of singularly disturbed nonlinear control dynamical systems
}

\author{
Byungik Kahng ${ }^{1}$ (D) \\ (c) Springer Nature Switzerland AG 2019
}

\begin{abstract}
We discuss the visualization algorithms and their justification theorems of the steady state sets of a class of nonlinear disturbed control dynamical systems. We focus upon the systems that include sudden large disturbance that is significant enough to cause a bifurcation (sudden change of the qualitative behavior) of the dynamics. We call such a disturbance, the singular disturbance. Under the singular disturbance, the traditional models of control theory generally fail to produce the desired point-convergence. Instead, the steady state sets often exhibit a fractal structure. The multiple valued iterative dynamics modeling proved to be a useful tool establishing the theoretical framework for this case. The main objective of this paper is to establish the theoretical foundation of this framework. First, we present our model and state the basic theorems that justify the algorithmic aspect of the model. Next, we prove the justification theorems. We use the iteration of the predecessor operators and the successor operators as the main tool. Afterwards, we discuss the usage of the justification theorems. We review the known visualization algorithms, with a due emphases on the usage of our main theorems.
\end{abstract}

Keywords Multiple valued iterative dynamics model · Disturbed control dynamical system · Maximal invariance . Invariant fractal

\section{Introduction}

\subsection{Introduction I: motivation and background information}

One of the two main objectives of the present research is to study the algorithmic and computational aspect of a class of nonlinear automatic control dynamical systems that are modeled by multiple valued iterative dynamical systems (MVIDS) [16]. We call such a model a multiple valued iterative dynamics model (MVID-Model) [16], and the corresponding computational algorithm, a multiple valued iterative dynamics algorithm (MVID-Algorithm) [15].

The MVID-modeling is useful characterizing and analyzing the steady state set and the long term behavior of a non-linear control dynamical system, when more traditional tools (such as Lyapunov stability theory) fail $[12,16]$. A typical failure takes place when a sudden large disturbance fundamentally changes the qualitative behavior of the system [15]. Such a phenomenon is commonly referred as a bifurcation. Here, "sudden" means the discontinuity with respect to time, and "large" refers to the magnitude bigger than the bifurcation point. See, for instance, [1] or [30], for more detail on the bifurcation theory in general.

It is well known that the bifurcation often generates the singularity (discontinuity and/or non-differentiability), and such bifurcations are abundant in nature. See, for instance, [6-9, 11-18, 20, 21], for this issue in various viewpoints. Due to the singularity, most of the traditional calculus-based models and techniques no longer work as

Byungik Kahng, byungik.kahng@untdallas.edu | ${ }^{1}$ Department of Mathematics and Information Sciences, University of North Texas at Dallas, 7400 University Hills Boulevard, Dallas, TX 75241, USA.

SN Applied Sciences (2019) 1:1061 | https://doi.org/10.1007/s42452-019-1110-3

Received: 9 January 2019 / Accepted: 16 August 2019 / Published online: 20 August 2019

SN Applied Sciences

A SPRINGER NATURE journal 
effectively, in such cases. The disturbance that causes the singularity is called the singular disturbance. A disturbed control dynamical system that includes the singular disturbance is called a singularly disturbed control dynamical system (SDCDS).

The particular topic we aim to study is the MVID model of SDCDS. The mathematical detail of this model will be elaborated in Sect. 2. Here in the introduction, we will have only a general overview through a limited visualization (Figs. 1, 2, 3). The illustrations in this paper are confined to $2 \mathrm{D}$ for easier visualization. Our MVID algorithms and the justification theorems, however, are stated for arbitrary finite dimension This is a standard treatment in control and automation theory, as in [25-29] and many others.

As exemplified by Figs. 1, 2 and 3, the steady state set of a MVID model of a SDCDS often exhibits a fractal structure. The analyses of the models and algorithms associated to these figures will be discussed in Sect. 4. See, also, [15, $17,20]$ for the elaborations in different perspectives. The emergence of the fractal structure is quite contrary to the traditional models of disturbed control dynamical system, in which case, the steady state set tend to be a single convex polytope, as exemplified by [25-29], for instance.

The fractal structure exemplified by Figs. 1, 2 and 3 is a natural consequence of the singular disturbance. Indeed, similar phenomena in nature have long been known and discussed in classical literature such as [24]. For MVID models of SDCDS, the fractal structure of Figs. 1, 2 and 3 is the result of a competition between multiple attracting points (imperfectly tuned multiple controllers) that are pushed away by (a) repelling point(s) in the interior (unstable target state). The applications of the singular disturbance closely related to this phenomenon include non-linear physics $[13,14,18]$, and digital signal processing [6-9]. In this paper, we consider a similar development in control and automation theory, partly as a continuation of [15, $16,20,21]$. See, also, [17] for a similar development in the viewpoint of an optimization research.
Fig. 1 Mutually interfering examples of the steady state sets of the MVID models of SDCDS's from [15]. The multifractal structure is generated by the interference of imperfectly tuned multiple controllers [15]
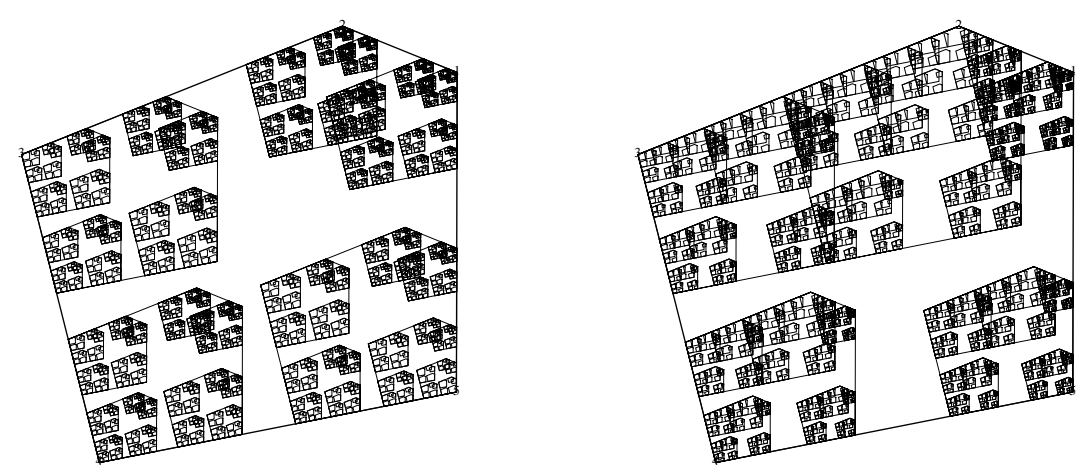

Fig. 2 Examples of the steady state sets of partially optimal SDCDS's under a non-interference condition from [19]. The non-interference condition certifies the simple fractal structure [17]

Fig. 3 Examples of the steady state sets of fully optimal SDCDS's under a non-interference condition from [17]. These show near-ideal cases
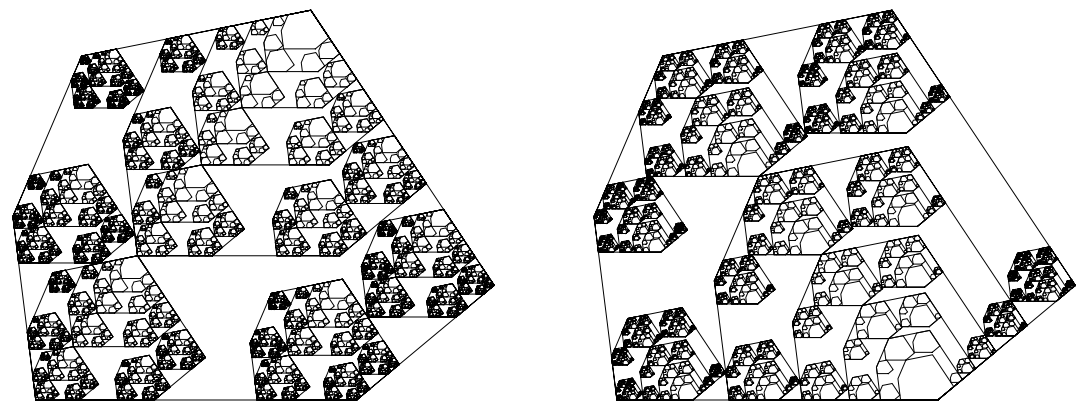
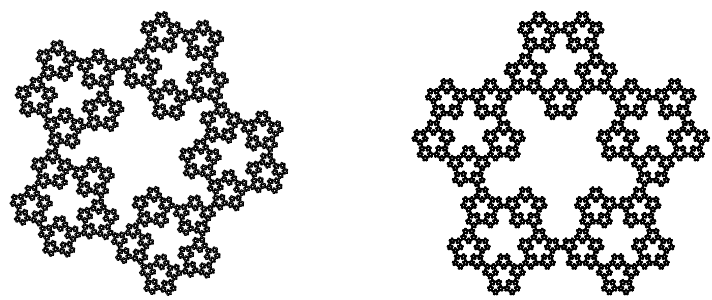


\subsection{Introduction II: main results}

This paper discusses the theory (Sects. 2 and 3 ) and the implementation (Sect. 4) of a class of MVID-models of SDCDS. The main contribution of this paper comes from the theory part. It consists of the justification theorems of the MVID algorithms, namely, Theorems 2.3, 2.7 and 2.8. These theorems literally justify the computational algorithms MVID models, as we will see in the implementation stage (Sect. 4).

Theorem 2.3 was completed in [16], so it will not be reproved here. The first original contribution of this paper is Theorem 2.7. It completes the preliminary works and the unsolved questions of [16], and provides the theoretical foundation of the numerical studies of [15] and [20]. The second main result is Theorem 2.8. It requires broader understanding on the fractal theory, and shares some key ideas with [17]. Prior reading of [17] will not be necessary, however. All technical details are established here, over the foundation of Theorem 2.7.

The implementation part of this paper is relatively minor. Its main purpose is to provide stronger motivation for proposing the MVID modeling and proving the justification theorems. Therefore, only a brief survey of analyses on known visualization algorithms will be discussed, with a due emphasis on the role of the justification theorems. Other aspects of the algorithmic research, such as optimization, will be discussed in a separate paper.

\section{Multiple valued iterative dynamics (MVID) modeling}

\subsection{MVID model}

A classical model of discrete-time non-linear disturbed control dynamical system (DCDS) can be written as follows, where the map $G$ is called the feedback control law, and the variables $v_{k}$ and $w_{k}$ represent the disturbance.

$\left\{\begin{array}{l}F:\left(x_{k}, u_{k}, w_{k}\right) \mapsto x_{k+1}, \\ G:\left(x_{k}, v_{k}\right) \mapsto u_{k} .\end{array}\right.$

The classical model assumes the continuity for each variable, and most of the time, negative Lyapunov exponents. It cannot handle, therefore, a sudden large disturbance, or a singular disturbance that includes the discontinuity (typically for $u, v$ or $w$ ). Also, as pointed out in [12] and [16], the classical model (2.1) is somewhat problematic in that it includes not only unknown but also unknowable variables. If all disturbance variables, say $u_{k}, v_{k}$ and $w_{k}$, could be determined, they would not have been disturbances to begin with. Partly to overcome this dilemma, and in another part to accommodate the singular disturbance, the author proposed a new approach, the multiple valued iterative dynamics model (MVID model) [10-12, 15, 16, 21]. Let us elaborate it as follows.

Definition 2.1 (Multiple Valued Iterative Dynamics Model [16]). Let $X, Y$ be non-empty sets, and $\mathscr{P}(X), \mathscr{P}(Y)$ be their power sets. We say a set function $f: \mathscr{P}(X) \rightarrow \mathscr{P}(Y)$ is a multiple valued map (function) from $X$ to $Y$ if

$f(S)=\bigcup\{f(x): x \in S\}$,

for all $S \subset X$. Here, $f(x)$ is the abbreviation of $f(\{x\})$. In particular, if $X=Y$, we call the dynamical system on $X$ given by the iteration of $f$ in $\mathscr{P}(X)$, the multiple valued iterative dynamical system (MVIDS). If an MVIDS was used to model a disturbed control dynamical system, we call such a model, a multiple valued iterative dynamics model (MVID Model).

In a sense, the MVID modeling is a generalization of the classical model (2.1) through the iterative dynamics of $f: x_{k} \mapsto\left\{F\left(x_{k}, G\left(x_{k}, v_{k}\right), w_{k}\right)\right\}$, for all possible or for all statistically meaningful disturbances captured by $v_{k}$ and $w_{k}$ values. See [12] for more detail on this generalization, including the proof of the validity theorem.

Remark 2.2 Besides the author's MVID modeling, alternative set-dynamics models were developed by other researchers such as [2] and [26]. The main ideas of all these models are more or less equivalent.

\subsection{Advantages of MVID model}

The advantages of the MVID modeling are twofold. First, it is well equipped to handle sudden large disturbances, or singular disturbances $[15,16]$, which most of the traditional models of disturbed control dynamics models fail to cover. See $[11,12,15,16]$ for the author's treatment on this issue. The singular disturbance is an important topic, because the bifurcation of the qualitative behavior of the dynamics due to the singular disturbance is a common occurrence in nonlinear physics. See $[13,14,17,18]$ for more detail on this connection.

The second advantage is the computational effectiveness. Because the MVID modeling does not add more variables, say $u_{k}, v_{k}$ and $w_{k}$ as in the classical DCDS model of (2.1), the algorithm can be made simpler and the 
computation quicker. [15] and [16] exemplify this issue via a comparison of computation-speed. See, also, [20] for a similar issue on a slightly different system.

\subsection{Maximal invariance in MVID model}

The controllability and the approximate control of maximal invariance are important topics in any nonlinear control and automation theory. For instance, a closed loop system [23] of an automatic control system runs in the maximal quasi-invariant set [21-23]. However, the concept of maximal invariance is a good deal more complicated in MVID models, compared to that of classical DCDS models.

Theorem 2.3 (Maximal Invariant Sets and Their Properties [16]). Let $X$ be a non-empty set, whose points we will call states. Let $Y$ be a non-empty subset of $X$, which represent a set of desirable states. Finally, let $f: \mathscr{P}(X) \rightarrow \mathscr{P}(X)$ be a multiple valued self map on $X$, whose iteration forms an MVIDS on $X$. We define the strong locally maximal quasiinvariant subset, $\mathcal{M}_{s}^{+}(Y)$, the weak locally maximal quasi-invariant subset, $\mathcal{M}_{w}^{+}(Y)$, the strong locally maximal full-invariant subset, $\mathcal{M}_{s}(Y)$, and the weak locally maximal full-invariant subset, $\mathcal{M}_{w}(Y)$ of $Y$ as follows.

$$
\begin{aligned}
& \mathcal{M}_{s}^{+}(Y)=\bigcup\{S \subset Y: \forall x \in S, f(x) \subset S\}, \\
& \mathcal{M}_{w}^{+}(Y)=\bigcup\{S \subset Y: \forall x \in S, f(x) \cap S \neq \emptyset\}, \\
& \mathcal{M}_{s}(Y)=\bigcup\{S \subset Y: \forall x \in S, x \in f(S) \text { and } f(x) \subset S\}, \\
& \mathcal{M}_{w}(Y)=\bigcup\{S \subset Y: \forall x \in S, x \in f(S) \text { and } f(x) \cap S \neq \emptyset\} .
\end{aligned}
$$

Then, $\mathcal{M}_{s}^{+}(Y)$ turns out to be the set of all states whose forward dynamics always stays in $Y$, while $\mathcal{M}_{w}^{+}(Y)$ is the set of all states whose forward dynamics can stay in $Y$ all the time. Also, $\mathcal{M}_{s}(Y)$ is the steady-state set of all dynamics that must always stay within $Y$, while $\mathcal{M}_{w}(Y)$ is the steady-state set of all possible dynamics within $Y$.

Proof See [16].

Remark 2.4 All maximal invariant sets mentioned in Theorem 2.3 are the optimal bounds in terms of the set inclusion. The strong maximal invariance (both quasi and full) is important in traditional robust control problem, while the weak maximal invariance is important for inverse problems. Many other cases such as adaptive control problems fall in between these two optimal cases.

Remark 2.5 Theorem 2.3 is, in fact, a superficial combination of a number of theorems in [16], re-written to fit the purpose of this paper. In [16], the concept of the "orbitchains" was used to characterize the maximal invariant sets. Here, most of the in-depth descriptions not directly relevant to our main topic were snipped out for brevity.

\subsection{The controllability theorem}

Now, let us turn ourselves to the controllability / reachability theorem, the main contribution of this paper. First, we begin with the following definition, which upgrades similar concepts of [16].

Definition 2.6 (Restricted Predecessor/Successor Operators). Let $X, Y$ and $f$ be as in Theorem 2.3. We define the set functions $\left(\left.f_{s}\right|_{Y}\right)^{-1}: \mathscr{P}(X) \rightarrow \mathscr{P}(Y)$ and $\left(\left.f_{w}\right|_{Y}\right)^{-1}: \mathscr{P}(X) \rightarrow \mathscr{P}(Y)$ as,

$\left\{\begin{array}{l}\left(\left.f_{s}\right|_{Y}\right)^{-1}(S)=\{y \in Y: f(y) \subset S\}, \\ \left(\left.f_{w}\right|_{Y}\right)^{-1}(S)=\{y \in Y: f(y) \cap S \neq \emptyset\} .\end{array}\right.$

We call the set functions $\left(\left.f_{s}\right|_{Y}\right)^{-1}$ and $\left(\left.f_{W}\right|_{Y}\right)^{-1}$, the strong predecessor operator of $f$ restricted to $Y$ and the weak predecessor operator of $f$ restricted to $Y$. Similarly, we restrict the forward application of $f$ to $Y$ as follows to create the set function $\left(\left.f\right|_{Y}\right): \mathscr{P}(X) \rightarrow \mathscr{P}(Y)$.

$\left(\left.f\right|_{Y}\right)(S)=Y \cap f(S)=\{y \in Y: y \in f(S)\}$.

We call $\left.f\right|_{Y}$, the successor operator of $f$ restricted to $Y$.

It turns out that $\left(\left.f_{W}\right|_{Y}\right)^{-1}$ and $\left(\left.f\right|_{Y}\right)$ are multiple valued maps (Definition 2.1), but $\left(\left.f_{s}\right|_{Y}\right)^{-1}$ is not. The proof is simple and left to the readers.

If $Y=X$, then the predecessor operators are said to be unrestricted, and we abbreviate them as $f_{s}^{-1}$ and $f_{w}^{-1}$, respectively. Using these abbreviations, we can re-write the equality (2.4) in the following form for all non-empty subset $Y$ of $X$.

$\left(\left.f_{s}\right|_{Y}\right)^{-1}(S)=Y \cap f_{S}^{-1}(S)$ and $\left(\left.f_{w}\right|_{Y}\right)^{-1}(S)=Y \cap f_{w}^{-1}(S)$.

Using the iterative dynamics of the restricted predecessor and successor operators, we can rigorously elaborate the preliminary findings of [16] as follows. 
Theorem 2.7 (Controllability Theorem). In addition to the conditions of Theorem 2.3, let us assume that both $f(y)$ and $f_{w}^{-1}(y)=\{x \in X: y \in f(x)\}$ are finite. Furthermore, let

$$
\begin{aligned}
\left(\left.f_{S}\right|_{Y}\right)^{-\infty}(S) & =\bigcap_{n=0}^{\infty}\left(\left.f_{S}\right|_{Y}\right)^{-n}(S), \\
\left(\left.f_{W}\right|_{Y}\right)^{-\infty}(S) & =\bigcap_{n=0}^{\infty}\left(\left.f_{W}\right|_{Y}\right)^{-n}(S), \\
\left(\left.f\right|_{Y}\right)^{\infty}(S) & =\bigcap_{n=0}^{\infty}\left(\left.f\right|_{Y}\right)^{n}(S) .
\end{aligned}
$$

Then, the following descending chain inequalities hold.

$$
\begin{aligned}
& Y \supset\left(\left.f_{s}\right|_{Y}\right)^{-1}(Y) \supset\left(\left.f_{s}\right|_{Y}\right)^{-2}(Y) \supset \cdots \supset\left(\left.f_{s}\right|_{Y}\right)^{-\infty}(Y) \\
& =\mathcal{M}_{s}^{+}(Y), \\
& Y \supset\left(\left.f_{w}\right|_{Y}\right)^{-1}(Y) \supset\left(\left.f_{w}\right|_{Y}\right)^{-2}(Y) \supset \cdots \supset\left(\left.f_{w}\right|_{Y}\right)^{-\infty}(Y) \\
& =\mathcal{M}_{w}^{+}(Y), \\
& Y \supset\left(\left.f\right|_{Y}\right)^{1} \circ\left(\left.f_{s}\right|_{Y}\right)^{-1}(Y) \supset \cdots \supset\left(\left.f\right|_{Y}\right)^{\infty} \circ\left(\left.f_{s}\right|_{Y}\right)^{-\infty}(Y) \\
& =\mathcal{M}_{s}(Y), \\
& Y \supset\left(\left.f\right|_{Y}\right)^{1}(Y) \cap\left(\left.f_{w}\right|_{Y}\right)^{-1}(Y) \supset \cdots \supset\left(\left.f\right|_{Y}\right)^{\infty}(Y) \\
& \quad \cap\left(\left.f_{w}\right|_{Y}\right)^{-\infty}(Y)=\mathcal{M}_{w}(Y) .
\end{aligned}
$$

That is, all maximal invariant sets are controllable (reachable) in a countably many steps.

Proof The proof will be presented in Sect. 3.1 of Sect. 3.

The finite step approximate control problem is meaningful only if the target set is reachable in a countable step. Through Theorem 2.7, we just established the the reachability of various maximal invariant sets (strong versus weak, and quasi versus full) under the finiteness condition (both $f(y)$ and $f_{w}^{-1}(y)$ are finite).

\subsection{The global attractor theorem}

While Theorem 2.3 established the initial set and the terminal set (steady-state set) in terms of the maximal invariance, Theorem 2.7 proved that those sets are controllable under the finiteness condition mentioned in the beginning of Theorem 2.7. However, these theorems contribute little toward the question whether the iterates of the sets (or the individual orbits of states) actually converge to the terminal set (steady state set). This is an essential step toward the full justification of the finite step numerical approximation problem, the topic for the next section.

The following theorem fills in this gap for our purpose. In fact, it completes the justification of the finite step approximation algorithm of our MVID model (Sect. 4.1). A simpler version of this theorem was done in [17]. It was for the problems on self-similar fractals and their dimension computation. For the purpose of this paper, we generalize it accordingly as follows.

Theorem 2.8 (Global Attractor Theorem). Let $v_{1}, \ldots, v_{N}$ be points in $\mathbb{R}^{n}$ such that they form the vertices of their convex hull $K\left(v_{1}, \ldots, v_{N}\right)$. For each $i \in\{1, \ldots, N\}$, let $f_{i}: \mathbb{R}^{n} \rightarrow \mathbb{R}^{n}$ be a contraction map with the contraction ratio (Lyapunov multiplier) $r_{i} \in(0,1)$ that fixes $v_{i} \in \mathbb{R}^{n}$. That is,

$d\left(f_{i}(x), f_{i}(y)\right) \leq r_{i} \cdot d(x, y)$ and $f_{i}\left(v_{i}\right)=v_{i}$.

Suppose further that $f_{i}\left(K\left(v_{1}, \ldots, v_{N}\right)\right) \subset K\left(v_{1}, \ldots, v_{N}\right)$. Finally, letf $: \mathscr{P}\left(\mathbb{R}^{n}\right) \rightarrow \mathscr{P}\left(\mathbb{R}^{n}\right)$ be a set function defined by,

$f(S)=f_{1}(S) \cup \cdots \cup f_{N}(S)$.

Then, every compact subset $Y$ of $\mathbb{R}^{n}$ that contains $K\left(v_{1}, \ldots, v_{N}\right)$ satisfies,

$\mathcal{M}_{s}(Y)=\mathcal{M}_{w}(Y)=\bigcap_{k=0}^{\infty} f^{k}(Y)=\bigcap_{k=0}^{\infty} f^{k}\left(K\left(v_{1}, \ldots, v_{N}\right)\right)$.

Moreover, the set given by the above equality is the global attractor in a sense that

$d\left(f^{m}(x), \bigcap_{k=0}^{\infty} f^{k}\left(K\left(v_{1}, \ldots, v_{N}\right)\right)\right) \rightarrow 0$

as $m \rightarrow \infty$, for any $x \in \mathbb{R}^{n}$. Here, $d$ represents the standard distance function in $\mathbb{R}^{n}$.

Proof The proof will be presented in Sect. 3.2 of Sect. 3 .

The following corollary is related to the interference between multiple controllers, and partly justifies the pattern formation of the interference-noise, a well known phenomenon that goes back to early 1970s [24]. 
Corollary 2.9 (Invariant Fractal). In addition to the conditions of Theorem 2.8, let us impose the non-interference condition given as follows.

$i \neq j \Longrightarrow \operatorname{int}\left(f_{i}\left(K\left(v_{1}, \ldots, v_{N}\right)\right) \cap f_{j}\left(K\left(v_{1}, \ldots, v_{N}\right)\right)\right)=\emptyset$.

Then, the steady state set, $\bigcap_{k=0}^{\infty} f^{k}\left(K\left(v_{1}, \ldots, v_{N}\right)\right)$ has a simple fractal structure. Moreover, if each $f_{i}$ is an affine or a piecewise affine contraction, then the steady state set is a self-similar fractal.

Proof This is an immediate corollary of Theorem 2.8, in terms of the construction of fractals, multi-fractals, and self-similar fractals. The equality (2.13), which corresponds to the non-interference in signal processing and control theory, is called the open set condition [5] in fractal geometry. See, for instance, [3] or [4] for detail.

\section{The proof of the justification theorems}

This is a technical section that concerns the proof of the justification theorems, Theorems 2.7 and 2.8. Theorem 2.3 is done throughly in [16], so we will not reproduce it here.

\subsection{The Proof of Theorem 2.7}

We divide the proof in three parts for easier reading.

The Proof of Theorem 2.7, Part I First, we prove the first, the second and the fourth descending chain inequalities of
Theorem 2.7. From the definitions of the restricted predecessor and successor operators, we can prove that these operators preserve the set inclusion as follows. Let $S \subset T$, and $y \in Y$. Then,

$y \in\left(\left.f_{s}\right|_{Y}\right)^{-1}(S) \Rightarrow f(y) \subset S \Rightarrow f(y)$

$$
\subset T \Rightarrow y \in\left(\left.f_{S}\right|_{Y}\right)^{-1}(T)
$$

$y \in\left(\left.f_{w}\right|_{Y}\right)^{-1}(S) \Rightarrow f(y) \cap S \neq \emptyset \Rightarrow f(y)$

$\cap T \neq \emptyset \Rightarrow y \in\left(\left.f_{w}\right|_{Y}\right)^{-1}(T)$,

$y \in\left(\left.f\right|_{Y}\right)(S) \Rightarrow y \in f(S) \Rightarrow y \in f(T) \Rightarrow y \in\left(\left.f\right|_{Y}\right)(T)$.

Therefore, repeatedly applying the restricted predecessor operators $\left(\left.f_{s}\right|_{Y}\right)^{-1},\left(\left.f_{w}\right|_{Y}\right)^{-1}$, and the restricted successor operator $\left(\left.f\right|_{Y}\right)$ to the initial set inequalities $Y \supset\left(\left.f_{S}\right|_{Y}\right)^{-1}(Y)$, $Y \supset\left(\left.f_{w}\right|_{Y}\right)^{-1}(Y)$, and $Y \supset\left(\left.f\right|_{Y}\right)(Y)$, we get the following descending chain inequalities.

$$
\left\{\begin{array}{l}
Y \supset\left(\left.f_{s}\right|_{Y}\right)^{-1}(Y) \supset\left(\left.f_{s}\right|_{Y}\right)^{-2}(Y) \supset \cdots \supset\left(\left.f_{s}\right|_{Y}\right)^{-\infty}(Y), \\
Y \supset\left(\left.f_{w}\right|_{Y}\right)^{-1}(Y) \supset\left(\left.f_{w}\right|_{Y}\right)^{-2}(Y) \supset \cdots \supset\left(\left.f_{w}\right|_{Y}\right)^{-\infty}(Y), \\
Y \supset\left(\left.f\right|_{Y}\right)^{1}(Y) \supset\left(\left.f\right|_{Y}\right)^{2}(Y) \supset \cdots \supset\left(\left.f\right|_{Y}\right)^{\infty}(Y) .
\end{array}\right.
$$

The first two chains of the set inequality (3.1) are the same as those of Theorem 2.7. Intersecting the second chain and the third chain term by term, we get the fourth chain of Theorem 2.7.

The third chain of Theorem 2.7 follows from the principal diagonal chain of the following diagram. This diagram consists of the first chain of the inequality (3.1) arranged vertically, and the iteration of the restricted successor operator arranged horizontally. Here, each arrow $(\rightarrow)$ stands for going down a descending set inclusion (つ).

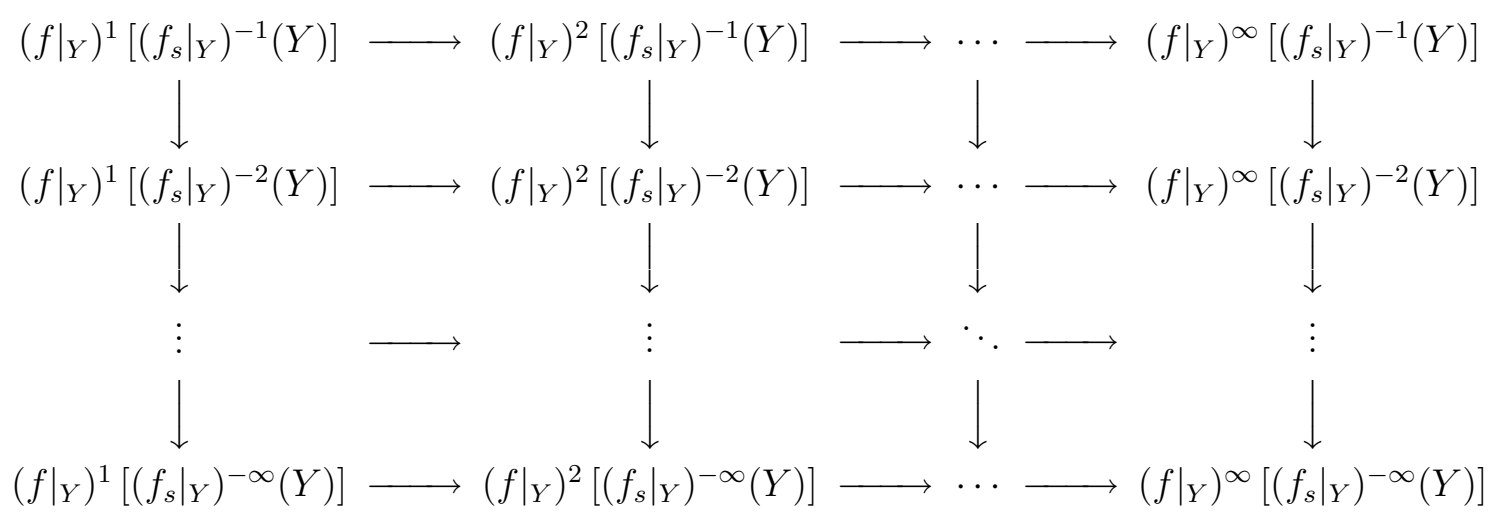


This completes the proof of the the descending chain part of Theorem 2.7.

The Proof of Theorem 2.7, Part Il The descending chain part of (2.8) is done. We must prove the final equalities. First, we prove the following inclusion.

$\left\{\begin{array}{l}\mathcal{M}_{s}^{+}(Y) \subset\left(\left.f_{s}\right|_{Y}\right)^{-\infty}(Y), \\ \mathcal{M}_{w}^{+}(Y) \subset\left(\left.f_{w}\right|_{Y}\right)^{-\infty}(Y), \\ \mathcal{M}_{s}(Y) \subset\left(\left.f\right|_{Y}\right)^{\infty}\left[\left(\left.f_{s}\right|_{Y}\right)^{-\infty}(Y)\right], \\ \mathcal{M}_{W}(Y) \subset\left(\left.f\right|_{Y}\right)^{\infty}(Y) \cap\left(\left.f_{w}\right|_{Y}\right)^{-\infty}(Y) .\end{array}\right.$

As described in Theorem 2.3, $\mathcal{M}_{s}^{+}(Y)$ consists of the states $x_{0} \in Y$, where any forward dynamics $\left(x_{k}\right)_{k=0}^{\infty}$, $x_{k} \in f\left(x_{k-1}\right), k \in\{1,2,3, \ldots\}$, of $x_{0}$ must stay entirely in $Y$, that is, $\forall x_{k} \in Y$, for any choice of forward orbit $\left(x_{k}\right)_{k=0}^{\infty}$. Therefore, $\forall x_{1} \in f\left(x_{0}\right), x_{1} \in Y$, thus yielding $f\left(x_{0}\right) \subset Y$, or $x_{0} \in\left(\left.f_{s}\right|_{Y}\right)^{-1}(Y)$. Similarly, $\forall x_{2} \in f^{2}\left(x_{0}\right)=f\left(f\left(x_{0}\right)\right) \subset Y$, or $x_{2} \in\left(\left.f_{s}\right|_{Y}\right)^{-1}\left(\left(\left.f_{s}\right|_{Y}\right)^{-1}(Y)\right)=\left(\left.f_{s}\right|_{Y}\right)^{-2}(Y)$. Repeating this process, we get, $x_{0} \in\left(\left.f_{s}\right|_{Y}\right)^{-k}(Y)$ for all $k \in\{1,2,3, \ldots\}$, and upon taking the intersection, we get $\mathcal{M}_{s}^{+}(Y) \subset\left(\left.f_{s}\right|_{Y}\right)^{-\infty}(Y)$.

On the other hand, $\mathcal{M}_{w}^{+}(Y)$ consists of the states $x_{0} \in Y$, which has at least one forward dynamics $\left(x_{k}\right)_{k=0}^{\infty}, x_{k} \in f\left(x_{k-1}\right), k \in\{1,2,3, \ldots\}$, so that $\forall x_{k} \in Y$. Here, $x_{1} \in f\left(x_{0}\right) \cap Y$, or $x_{0} \in\left(\left.f_{w}\right|_{Y}\right)^{-1}(Y)$. Similarly, $x_{2} \in f\left(x_{1}\right) \cap Y$. This condition, coupled with $x_{1} \in f\left(x_{0}\right) \cap Y$, yield $x_{0} \in\left(\left.f_{w}\right|_{Y}\right)^{-2}(Y)$. Repeating this process, we get $x_{0} \in\left(\left.f_{w}\right|_{Y}\right)^{-k}(Y)$ for all $k \in\{1,2,3, \ldots\}$, and upon taking the intersection, we get $\mathcal{M}_{w}^{+}(Y) \subset\left(\left.f_{w}\right|_{Y}\right)^{-\infty}(Y)$.

From the definitions of the maximal full invariance in the equality (2.3), one can clearly see that $\mathcal{M}_{s}(Y) \subset \mathcal{M}_{s}^{+}(Y) \subset\left(\left.f_{s}\right|_{Y}\right)^{-\infty}(Y)$ and $\mathcal{M}_{w}(Y) \subset \mathcal{M}_{w}^{+}(Y) \subset\left(\left.f_{w}\right|_{Y}\right)^{-\infty}(Y)$. Thus, from the strong/weak local invariance of $\mathcal{M}_{s}(Y)$ and $\mathcal{M}_{w}(Y)$, we get $\mathcal{M}_{s}(Y)=\left(\left.f\right|_{Y}\right)\left(\mathcal{M}_{s}(Y)\right)=\left(\left.f\right|_{Y}\right)^{2}\left(\mathcal{M}_{s}(Y)\right)=\cdots=\left(\left.f\right|_{Y}\right)^{\infty}$ $\left(\mathcal{M}_{s}(Y)\right) \subset\left(\left.f\right|_{Y}\right)^{\infty}\left[\left(\left.f_{s}\right|_{Y}\right)^{-\infty}(Y)\right]$, and $\mathcal{M}_{w}(Y) \subset\left(\left.f\right|_{Y}\right)^{\infty}$ $\left(\mathcal{M}_{w}(Y)\right) \cap\left(\left.f_{w}\right|_{Y}\right)^{-\infty}(Y) \subset\left(\left.f\right|_{Y}\right)^{\infty}(Y) \cap\left(\left.f_{w}\right|_{Y}\right)^{-\infty}(Y)$.

The Proof of Theorem 2.7, Part III The final step is to prove the other direction of the inclusion. That is,

$\begin{cases}\mathcal{M}_{s}^{+}(Y) & \supset\left(\left.f_{s}\right|_{Y}\right)^{-\infty}(Y), \\ \mathcal{M}_{w}^{+}(Y) & \supset\left(\left.f_{w}\right|_{Y}\right)^{-\infty}(Y), \\ \mathcal{M}_{s}(Y) & \supset\left(\left.f\right|_{Y}\right)^{\infty}\left[\left(\left.f_{s}\right|_{Y}\right)^{-\infty}(Y)\right], \\ \mathcal{M}_{w}(Y) & \supset\left(\left.f\right|_{Y}\right)^{\infty}(Y) \cap\left(\left.f_{w}\right|_{Y}\right)^{-\infty}(Y) .\end{cases}$

Recall that we assumed the finiteness condition, that is, both $f(y)$ and $f_{w}^{-1}(y)$ are finite sets. It turns out, however, the first inclusion of (3.3) holds in general, without the finiteness condition. The proof of the other inclusions depend upon the finiteness condition.

Given $x_{0} \in\left(\left.f_{s}\right|_{Y}\right)^{-\infty}(Y)$, choose any forward orbit $\left(x_{k}\right)_{k=0^{\prime}}^{\infty}$ $x_{k} \in f\left(x_{k-1}\right), k \in\{1,2,3, \ldots\}$. Then, $\forall x_{k} \in Y$, because $x_{0} \in\left(\left.f_{s}\right|_{Y}\right)^{-\infty}(Y) \subset\left(\left.f_{s}\right|_{Y}\right)^{-k}(Y)$. Hence, $x_{0} \in \mathcal{M}_{s}^{+}(Y)$, by Theorem 2.3. This proves the first inclusion, $\left(\left.f_{s}\right|_{Y}\right)^{-\infty}(Y) \subset \mathcal{M}_{s}^{+}(Y)$.

To prove the second inclusion, choose any $y_{0} \in\left(\left.f_{w}\right|_{Y}\right)^{-\infty}(Y)$. That is, $y_{0} \in\left(\left.f_{w}\right|_{Y}\right)^{-n}(Y)$ for all $n \in\{1,2,3, \ldots\}$, or equivalently, $x_{0}, x_{1}, \ldots, x_{n} \in Y$ for some forward orbit $\left(x_{k}\right)_{k=0^{\prime}}^{n} x_{k} \in f\left(x_{k-1}\right), k \in\{1,2, \ldots, n\}$ and $x_{0}=y_{0}$. This holds for all $n$ 's, which define infinitely many forward orbits $\left(x_{k}\right)_{k=0^{\prime}}^{n} n \in\{1,2,3, \ldots\}$. Because $f\left(y_{0}\right)$ is finite, this process cannot generate infinitely many $x_{1}$ 's. Instead, a certain $x_{1}$ must be shared by infinitely many forward orbit chains. Let us fix this $x_{1}$ and name it $y_{1} \in Y$. Then, by this construction, there are infinitely many forward orbits of $y_{0}$ that go through $y_{1}$. We apply the finiteness condition (this time, $f\left(y_{1}\right)$ is finite) to the orbits that begin with $y_{0}$ and then $y_{1}$. By the same argument, we get $y_{2} \in Y$, such that there are infinitely many forward orbits of $y_{0}$ that go through $y_{0}, y_{1}$, and then, $y_{2}$. Repeating this process, we get the forward orbit $\left(y_{k}\right)_{k=0}^{\infty}$ that stays within $Y$ entirely. Hence, $y_{0} \in \mathcal{M}_{w}^{+}(Y)$, by Theorem 2.3 , consequently yielding the second inclusion, $\left(\left.f_{w}\right|_{Y}\right)^{-\infty}(Y) \subset \mathcal{M}_{w}^{+}(Y)$.

The fourth inclusion is based on the same idea as the above, thus relying upon the finiteness condition. Chose any $y_{0} \in\left(\left.f\right|_{Y}\right)^{\infty}(Y) \cap\left(\left.f_{w}\right|_{Y}\right)^{-\infty}(Y)$. Because $y_{0} \in\left(\left.f_{w}\right|_{Y}\right)^{-\infty}(Y)$, there exists a certain forward orbit $\left(y_{k}\right)_{k=0}^{\infty}$ of $y_{0}, y_{k} \in f\left(x_{k-1}\right), k \in\{1,2,3, \ldots\}$ within $Y$. Also, from $y_{0} \in\left(\left.f\right|_{Y}\right)^{\infty}(Y)$, for any $n \in\{1,2,3, \ldots\}$, there exists a certain $x_{-n} \in Y$ such that the orbit $\left(x_{k}\right)_{k=-n^{\prime}}^{0} x_{k} \in f\left(x_{k-1}\right)$, $x_{0}=y_{0}$ within $Y$. Applying the same finiteness argument backward, we get a backward orbit $\left(y_{k}\right)_{k \rightarrow-\infty}^{0}$ in $Y$, and consequently, a doubly infinite orbit $\left(y_{k}\right)_{k \rightarrow-\infty}^{\infty}$ in $Y$. Setting $S=\left\{y_{k}\right\}_{k \rightarrow-\infty}^{k \rightarrow \infty}$, we can see that $S$ includes $y_{0}$ and and satisfies the condition for $\mathcal{M}_{w}(Y)$ of (2.3).

The third inclusion is based upon the fact that $\left(\left.f_{s}\right|_{Y}\right)^{-\infty}(Y)=\mathcal{M}_{s}^{+}(Y)$, which we proved in the second paragraph. Because $\mathcal{M}_{s}^{+}(Y)$ is strongly quasi-invariant under $\left.f\right|_{Y}$, we can restrict ourselves to the iterative dynamics of the self map, $\left.f\right|_{Y}: \mathcal{M}_{s}^{+}(Y) \rightarrow \mathcal{M}_{s}^{+}(Y)$. Therefore, we can apply the known results for global maximal invariance studied in [21]. Since $f$ is finite-to-one in entire $X$, so is $\left.f\right|_{\gamma}$. Hence, the third inclusion follows as in [21].

\subsection{The Proof of Theorem 2.8}

This time, we divide the proof in four parts.

The Proof of Theorem 2.8, Part I First, we prove the maximal invariance of the convex hull. Let $K=K\left(v_{1}, \ldots, v_{N}\right)$. Because $K$ is quasi-invariant under each $f_{i}$, it is quasi-invariant under $f$, too. Therefore, it can be regarded as the whole-space of the iterative MVID of $f$. Applying this to the equality (2.6), we get 
$\left\{\begin{array}{l}\left(\left.f_{s}\right|_{K}\right)^{-1}(K)=K \cap f_{s}^{-1}(K)=K \\ \left(\left.f_{w}\right|_{K}\right)^{-1}(K)=K \cap f_{w}^{-1}(K)=K .\end{array}\right.$

Repeating the iteration arbitrarily many times, we get the following conclusion.

$\left(\left.f_{s}\right|_{K}\right)^{-n}(K)=\left(\left.f_{w}\right|_{K}\right)^{-n}(K)=K$,

for any $n \in\{0,1,2, \ldots\}$. Finally, applying Theorem 2.7, we conclude,

$\mathcal{M}_{s}(K)=\mathcal{M}_{w}(K)=\bigcap_{k=0}^{\infty} f^{k}(K)$

The Proof of Theorem 2.8, Part II Next, we prove the last equality of (2.11). Because $K \subset Y$, we must have

$\bigcap_{k=0}^{\infty} f^{k}(K) \subset \bigcap_{k=0}^{\infty} f^{k}(Y)$

Let us prove the other direction. Choose any $y_{0} \in \bigcap_{k=0}^{\infty} f^{k}(Y)$. Then, for any $k \in\{1,2,3, \ldots\}$,

$y_{0}=f_{i_{1}} \circ \cdots \circ f_{i_{k}}\left(y_{-k}\right)$,

for some $i_{1}, \ldots, i_{k} \in\{1, \ldots, N\}$ and $y_{-k} \in Y$.

Observe that $\bigcap_{k=0}^{\infty} f^{k}(K)$ is a nonempty compact set, because it is a nested intersection of compact sets. Therefore, we can select an element $x_{-k} \in \bigcap_{k=0}^{\infty} f^{k}(K)$. Because $K$ is strongly invariant, as we discussed in the first part of the proof,

$x_{0}=f_{i_{1}} \circ \cdots \circ f_{i_{k}}\left(x_{-k}\right) \in \bigcap_{k=0}^{\infty} f^{k}(K)$.

Here, $i_{1}, \ldots, i_{k}$ are the same indices as in the equality (3.6).

Let $\rho=\max \left\{r_{1}, \ldots, r_{N}\right\} \in(0,1)$. Then, because each $f_{i}$ is a contraction with the contraction ratio $r_{i} \leq \rho<1$, we get the following result from the equalities (3.6) and (3.7).

$$
\begin{aligned}
& d\left(y_{0}, \bigcap_{k=0}^{\infty} f^{k}(K)\right) \\
& \leq d\left(y_{0}, x_{0}\right) \\
& \leq r_{i_{1}} d\left(y_{-1}, x_{-1}\right) \\
& \cdots \\
& \leq\left(r_{i_{1}} \cdots r_{i_{k}}\right) \cdot d\left(y_{-k}, x_{-k}\right) \\
& \leq \rho^{k} \cdot \operatorname{diam}(X) .
\end{aligned}
$$

Here, $k$ is an arbitrary positive integer and $0<\rho<1$. Thus, we conclude

$d\left(y_{0}, \bigcap_{k=0}^{\infty} f^{k}(K)\right)=0$.

Now, recall that $\bigcap_{k=0}^{\infty} f^{k}(K)$ is compact. Therefore, the equality (3.8) implies,

$y_{0} \in \bigcap_{k=0}^{\infty} f^{k}(K)$.

Because $y_{0}$ was selected arbitrarily in $Y$, we get the following conclusion.

$\bigcap_{k=0}^{\infty} f^{k}(Y) \subset \bigcap_{k=0}^{\infty} f^{k}(K)$

Combining the set inequalities (3.5) and (3.9), we get the desired equality,

$\bigcap_{k=0}^{\infty} f^{k}(Y)=\bigcap_{k=0}^{\infty} f^{k}(K)$
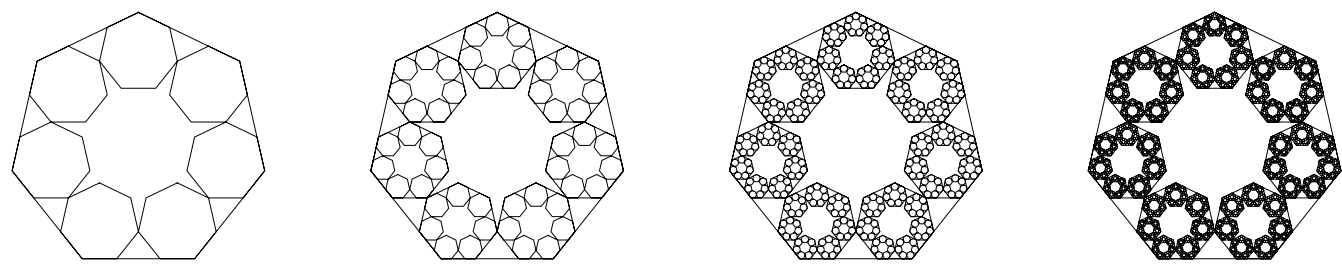

Fig. 4 An example of a forward iteration of an MVID Algorithm for a class of Nonlinear SDCDS Modeling $(N=7)$ 

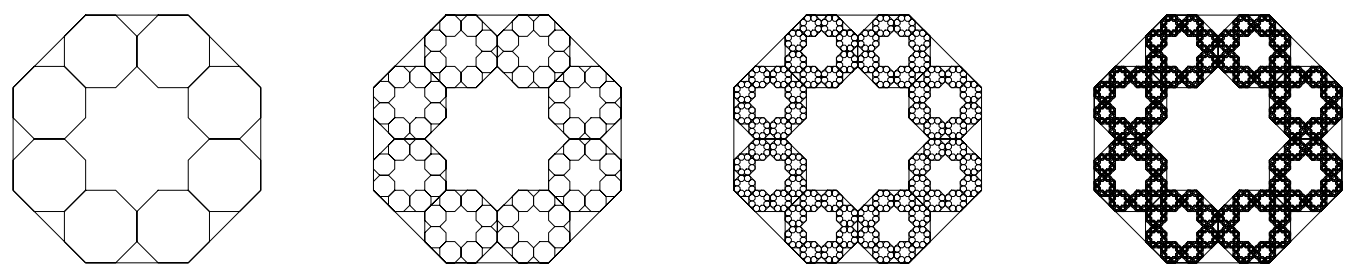

Fig. 5 An example of a forward iteration of an MVID Algorithm for a class of Nonlinear SDCDS Modeling $(N=8)$

The Proof of Theorem 2.8, Part III The third part is the maximality. Because $K \subset Y, \mathcal{M}_{s}(K) \subset \mathcal{M}_{s}(Y)$ and $\mathcal{M}_{w}(K) \subset \mathcal{M}_{w}(Y)$. We must prove the other direction. From Theorem 2.7, $\mathcal{M}_{s}(Y)=\left(\left.f\right|_{Y}\right)^{\infty} \circ\left(\left.f_{s}\right|_{Y}\right)^{-\infty}(Y)$ and $\mathcal{M}_{w}(Y)=\left(\left.f\right|_{Y}\right)^{\infty}(Y) \cap\left(\left.f_{w}\right|_{Y}\right)^{-\infty}(Y)$. Removing the restriction from the restricted predecessor/successor operators, we get the following ascending chains.

$$
\begin{aligned}
\mathcal{M}_{s}(Y)= & \left(\left.f\right|_{Y}\right)^{\infty} \circ\left(\left.f_{s}\right|_{Y}\right)^{-\infty}(Y) \subset\left(\left.f\right|_{Y}\right)^{\infty}(Y) \\
& \subset\left(\left.f\right|_{\mathbb{R}^{n}}\right)^{\infty}(Y), \\
\mathcal{M}_{w}(Y)= & \left(\left.f\right|_{Y}\right)^{\infty}(Y) \cap\left(\left.f_{w}\right|_{Y}\right)^{-\infty}(Y) \subset\left(\left.f\right|_{Y}\right)^{\infty}(Y) \\
& \subset\left(\left.f\right|_{\mathbb{R}^{n}}\right)^{\infty}(Y) .
\end{aligned}
$$

Applying the equalities (2.7) and (3.10) to the last quantity, we get,

$$
\begin{aligned}
& \mathcal{M}_{s}(Y) \subset \bigcap_{k=0}^{\infty} f^{k}(Y)=\bigcap_{k=0}^{\infty} f^{k}(K)=\mathcal{M}_{s}(K), \\
& \mathcal{M}_{w}(Y) \subset \bigcap_{k=0}^{\infty} f^{k}(Y)=\bigcap_{k=0}^{\infty} f^{k}(K)=\mathcal{M}_{w}(K) .
\end{aligned}
$$

This completes the proof of the equality (2.11).

The Proof of Theorem 2.8, Part IV The final piece is the global attraction. Given $x \in \mathbb{R}^{n}$, put $Y=K\left(x, v_{1}, \ldots, v_{N}\right)$. Then, the desired result (2.12) follows immediately from the equality (2.11).

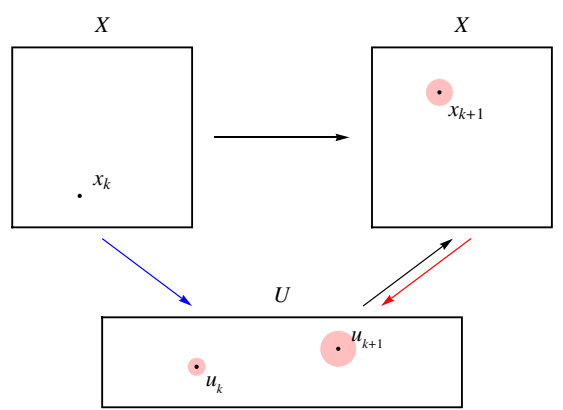

Fig. 6 Non-singular disturbance within MVID model [16]

\section{The implementation of the MVID algorithms}

The goal of this section is to provide further motivation for our theoretical research and main theorems. We exemplify and analyze the usage of Theorems 2.7 and 2.8 in the implementation stage of the MVID algorithms.

\subsection{The finite step approximate control}

Let us begin with the simplest examples. Figures 4 and 5 depict the implementation of the equality (2.11). Both examples show the case when the same controllers are placed symmetrically around a repeller. They illustrate, from the left to right, $\bigcap_{k=0}^{k=1} f^{k}(K), \bigcap_{k=0}^{k=2} f^{k}(K), \bigcap_{k=0}^{k=3} f^{k}(K)$ and $\bigcap_{k=0}^{k=5} f^{k}(K)$. Or equivalently, as in Theorem 2.7,

$\bigcap_{k=0}^{k=1} f^{k}(K) \supset \bigcap_{k=0}^{k=2} f^{k}(K) \supset \bigcap_{k=0}^{k=3} f^{k}(K) \supset \cdots \supset \bigcap_{k=0}^{k=5} f^{k}(K)$.

Due to the equalities (2.11) and (2.12), we can use $\bigcap_{k=0}^{k=n} f^{k}(K)$ of the descending chain (4.1) as a finitestep approximation of the maximal invariant sets, $\mathcal{M}_{s}(Y)$ or $\mathcal{M}_{w}(Y)$, for any compact set $Y$ that includes $K=K\left(v_{1}, \ldots, v_{N}\right)$ (Theorem 2.8). The fourth pictures (from the left) in Figs. 4 and 5 depict this finite-step approximation. Also, Figs. 1 and 2 in the introduction show similar finite-step approximations of the maximal invariant sets (only the maximal invariant sets, no descending chains).

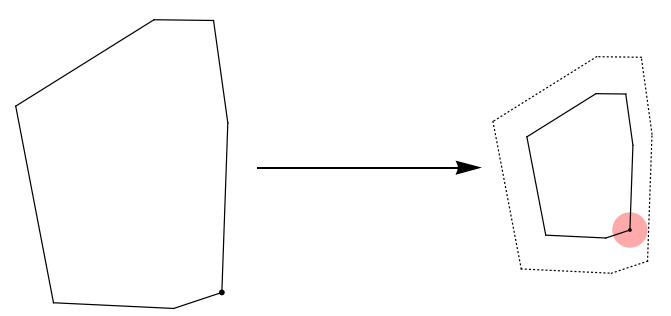



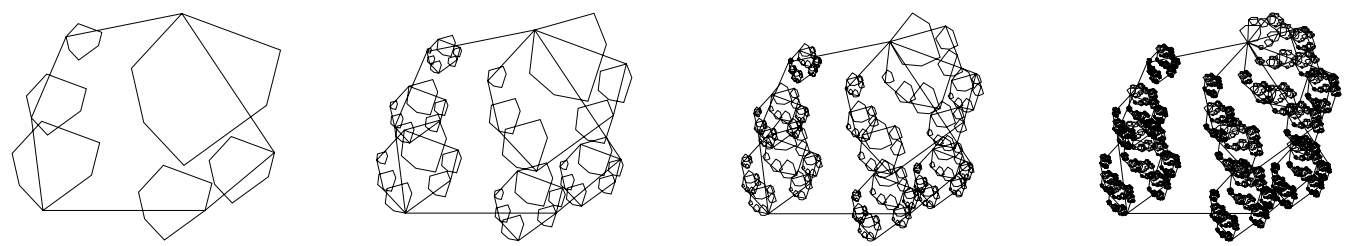

Fig. 7 An example of a forward iteration of an MVID Algorithm for a class of Nonlinear SDCDS Modeling, when a rotation component is added

Another implication of Theorem 2.8 is the speed of the attraction. The maximal Lyapunov multiplier of $f_{1}, \ldots, f_{N}$ is $\bar{r}=\max \left\{r_{1}, \ldots, r_{N}\right\}$, and the condition of Theorem 2.8 includes, $0<\bar{r}<1$. Hence, the order of attraction is exponential. For systems with small Lyapunov exponents as in Fig. 4 ( $\bar{r} \approx 0.307979)$ and in Fig. $5(\bar{r} \approx 0.292893)$, one can get a reasonably good approximation with only a small number of iteration ( $n=5$ for both cases). The same is true for Figs. 1 and 2.

\subsection{The non-interference condition}

Another important feature of of Figs. 4 and 5 is the optimality. Figures 4 and 5 illustrate the MVID models with the largest possible contraction ratio (Lyapunov multiplier) for given number of controllers, under the non-interference condition (2.13) of Corollary 2.9. See [17] for detail.

Upon relaxing the optimality condition a little, we get non-symmetric examples as in Fig. 2. The lower bound of the Lyapunov multiplier is the ratio between the diameter of the smallest subpolygon and that of the original polygon. One can clearly see that the ratio is much smaller for the examples of Fig. 2 compared to that of the symmetric cases (Figs. 4 and 5). See [17] for the proof.

What if we go further and lift the non-interference condition (2.13) too? The consequence is illustrated in Fig.1 as the overlapping subpolygons. In this case, the optimization of Lyapunov multiplier is meaningless. With no concern on the overlap and/or competitions from the nearby controllers, one can take the Lyapunov multipliers as large as desired, at worst even $\bar{r}>1$. Clearly, this is not a realistic model of well-functioning automatic control, and its failure justifies the relevance of the non-interference condition. See [15] for detail.

\subsection{The quasi-invariance condition}

One imperfection of this paper is the quasi-invariance condition of the convex hull $K\left(v_{1}, \ldots, v_{N}\right)$ in Theorem 2.8 . That is,

$f_{i}\left(K\left(v_{1}, \ldots, v_{N}\right) \subset K\left(v_{1}, \ldots, v_{N}\right), \quad i \in\{1, \ldots, N\}\right.$.
For the purpose of the MVID modeling that we had been considering so far, this restriction was appropriate. Figure 6 depict the justification. The first picture illustrates the effect of the disturbance variables $v_{k}$ and $w_{k}$ of the classical DCDS model (2.1), when they are applied as white noise. The second picture shows the aforementioned white noise being captured within a MVID model with slightly weaker contraction, or slightly higher Lyapunov multiplier. See [16] for detail.

Figure 7, on the other hand, depict a fundamentally different example. Here, the disturbance includes a significant and consistent rotation component, and thus the quasi-invariance condition (4.2) fails to apply. Note that the forward iterates of convex hull $K$ are not bounded within $K$ in Fig. 7. The second and the third pictures of Fig. 3 illustrate the same situation, only simpler and more optimal.

As shown in Figs. 3 and 7, this change creates an interesting twist to our problem that is worth studying as a future project. The fractal structure of the limit set is not too difficult to characterize. As for the possible maximal invariance and the controllability, I do not have complete solution as of this moment. I do conjecture affirmatively, and plan to develop the current research beyond this restriction.

\section{Conclusion}

Through this paper, we studied the theory and implementation of MVID modeling for a class of simple singularly disturbed nonlinear control dynamical systems. The original contribution of this paper was mainly on the theory part. We presented three justification theorems for our algorithmic research and the finite step approximate control of the steady state sets (terminal sets). The first (Theorem 2.3) was a summary of results from the author's earlier publication [16]. The second (Theorem 2.7) and the third (Theorem 2.8) were new, original contributions of this paper.

In terms of the implementation, we focused upon two aspects. The visualization (Figs. 1, 2, 3, 4, 5, 7), and the 
justification (Sect. 4.1). We discussed, also, the role of the non-interference condition (2.13) of Corollary 2.9, and its connection to the optimization of Lyapunov multipliers.

There is still some work to be done beyond the present research. As mentioned in Sect. 4.3, one may be able to generalize Theorem 2.8 further by going beyond the quasiinvariance condition (4.2). Numerical experiments support this conjecture (Fig. 7, for instance). For now, this issue is left as a possible future research project.

\section{Compliance with ethical standards}

Conflict of interest The author declares that he has no conflict of interest.

Human or animal participant The author declares that there was no human or animal participant involved in this research.

\section{References}

1. Arrowsmith DK, Place CM (1990) An introduction to dynamical systems. Wiley, Hoboken

2. Arstein Z, Rakovic S (2008) Feedback and invariance under uncertainty via set-iterates. Automatica 44:520-525

3. Edgar G (2007) Measure, topology, and fractal geometry, undergraduate texts in mathematics. Springer, Berlin

4. Falconer K (2003) Fractal geometry: mathematical foundations and application, 2nd edn. Wiley, Hoboken

5. Hutchinson J (1981) Fractals and self-similarity. Indiana J Math 30:713-747

6. Kahng B (2002) Dynamics of symplectic piecewise elliptic rotation maps on tori. Ergod Theory Dyn Syst 22:483-505

7. Kahng B (2004) Dynamics of kaleidoscopic maps. Adv Math 185:178-205

8. Kahng B (2004) The invariant fractals of symplectic piecewise affine elliptic dynamics. AMS Proc Symp Pure Math 72:375-389

9. Kahng B (2004) The unique ergodic measure of symmetric piecewise toral isometry with the rotation angle $\mathrm{pi} / 5$ is the hausdorff measure of its singular set. Dyn Syst 19:245-264

10. Kahng B (2008) The invariant set theory of multiple valued iterative dynamical systems. Recent Adv Syst Sci Simul Eng 7:19-24

11. Kahng B (2008) Maximal invariant sets of multiple valued iterative dynamics in disturbed control systems. Int J Circ Syst Signal Proc 2:113-120

12. Kahng B (2009) Positive invariance of multiple valued iterative dynamical systems in disturbed control models. In: Proceedings of mediterranean control conference. Thessaloniki, Greece

13. Kahng B (2009) Redifining chaos: Devaney-chaos for piecewise isometric dynamical systems. Int J Math Models Methods Appl Sci 4:317-326
14. Kahng B (2009) Singularities of 2-dimensional invertible piecewise isometric dynamics. Chaos 19:023115

15. Kahng $B$ (2010) The approximate control problems of the maximal invariant sets of non-linear discrete-time disturbed control dynamical systems: an algorithmic approach. In: Proceedings of 9th international conference on control automotive and systems (ICCAS), KINTEX, Gyeonggi-do, Korea

16. Kahng B (2013) Multiple valued iterative dynamics models of nonlinear discrete-time control dynamical systems with disturbance. J Korean Math Soc 50:17-39

17. Kahng $B$ (2016) An optimization of maximal invariance in a class of multiple valued iterative dynamics models of nonlinear disturbed control systems. Fractals 24:1650044

18. Kahng B, Cuadros M, Sullivan J (2014) Sliding singularities of bounded invertible planar piecewise isometric dynamics. Int J Math Models Methods Appl Sci 8:57-64

19. Kahng B, Gomez M, Padilla E Maximal dimensions of outer sierpinski fractals with a uniform contraction ratio, Submitted to Fractals

20. Kahng B, Gomez M, Padilla E (2016) Visualization algorithms for the steady state sets of a class of singularly disturbed nonlinear control dynamical systems. Int J Math Models Methods Appl Sci 10:237-243

21. Kahng B, Mendes M (2013) The characterization of maximal invariant sets of non-linear discrete control dynamical systems. Discret Contin Dyn Syst S: 393-406

22. Kerrigan E, Lygeros J, Maciejowski J (2002) A geometric approach to reachability computations for constrained discretetime systems, In: Proceedings of 15th IFAC World congress, Barcelona, Spain

23. Kerrigan E, Maciejowski J (2000) Invariant sets for constrained nonlinear discrete-time systems with application to feasibility in model predictive control, In: Proceedings of 39th IEEE conference on decision and control, Sydney, Australia

24. Mandelbrot B (1982) The fractal geometry of nature. W. H. Freeman, Holtzbrinck

25. Mayne DQ, Seron M, Rakovic SV (2005) Robust model predictive control of constrained linear systems with bounded disturbances. Automatica 41:219-224

26. Rakovic S, Gielen R (2014) Positively invariant families of sets for interconnected and time-delay discrete-time systems. SIAM J Control Opt 52:2261-2283

27. Rakovic SV, Kerrigan EC, Kouramas Kl, Mayne DQ (2005) Invariant approximations of the minimal robustly positively invariant sets. IEEE Trans Autom Control 50:406-410

28. Rakovic SV, Kerrigan EC, Mayne DQ, Kouramas KI (2007) Optimized robust control invariance for linear discrete-time systems: theoretical foundations. Automatica 43:831-841

29. Rakovic SV, Kerrigan EC, Mayne DQ, Lygeros J (2006) Reachability analysis of discrete-time systems with disturbances. IEEE Trans Autom Control 51:546-561

30. Wiggins S (2003) Introduction to applied nonlinear dynamical systems and chaos, texts in applied mathematics, 2 nd edn. Springer, Berlin

Publisher's Note Springer Nature remains neutral with regard to jurisdictional claims in published maps and institutional affiliations. 